\title{
A Fractionally Spaced DFE With Subband Decorrelation
}

\author{
Stephan Weiss ${ }^{1}$, Markus Rupp ${ }^{2}$, and Lajos Hanzo ${ }^{1}$ \\ ${ }^{1}$ Dept. Electronics \& Computer Science, University of Southampton, UK \\ ${ }^{2}$ Wireless Research Lab / Bell-Labs, Lucent Technologies, Holmdel, NJ, USA \\ $\{$ sw1,Ih\}@ecs.soton.ac.uk, rupp@lucent.com
}

\begin{abstract}
In this paper we proposed a modification of the "classic" fractionally spaced decision feedback equaliser (FS$D F E$ ) in order to increase the slow convergence rate of the oversampled feed-forward section for LMS type algorithms. This is performed by employing a subband structure for the latter part of the FS-DFE, which results in a decorrelation of the input. We motivate why an oversampled subband decomposition is beneficial and comment on the selection of the filter banks. As an additional benefit, computational savings arise if judicious implementation of the filter banks is combined with the internal decimation and expansion operations in the FS-DFE. Simulations for a sever multipath environment are presented.
\end{abstract}

\section{Introduction}

Fractionally spaced decision feedback equalisers (FS-DFE) based on LMS-type algorithms have recently been shown to exhibit considerably slower convergence than their symbol-spaced counterparts, particularly at high SNR [1]. This has been attributed to large eigenvalue spread of the equaliser's input signal. Since particularly in the area of broadband radio and TV over fixed wireless channels efforts go toward longer equalisation filters with additionally inhibited convergence speed, improvements of the convergence rate are generally desirable.

The main task of the equaliser is to retrieve a transmitted signal $u[n]$ from a distorted received copy $x[n]$ at the output of a channel $c[n]$, as given in Fig. 1. The

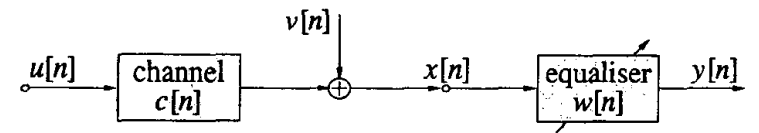

Fig. 1. Arrangement of channel and equaliser. equaliser $w[n]$, which may be non-linear due to an incorporated decision device, will try to reconstruct a signal $y[n]=u[n-\Delta]$, which is identical to the transmitted sequence of symbols $u[n]$ apart from a delay $\Delta$.

The general structure of an FS-DFE is shown in Fig. 2. It is based on an equation error IIR filter [2], with a feed-forward (FF) section processing the input signal $x[n]$, and a feedback (FB) section fed with the desired signal $d[n]$. In training mode (switch position 1 ), this signal $d[n]$ is a delayed version of the channel input, which in practise is achieved by transmitting a known training sequence. In data or decision feedback mode (switch position 2), it is assumed that the equaliser has been sufficiently adapted during training. If changes in the channel take place, the error $e[n]$ will increase and can be used to re-adapt and track the nonstationarity. The non-stationarities have to be slow enough such that the equaliser adaptation can follow.

The FF section $a[n]$ of the equaliser is generally dedicated to the cancellation of pre-cursor inter-symbol interference (ISI), or the maximum phase part of the channel. The inversion of the minimum phase part of the channel or post-cursor ISI cancellation is the task of the FB part $b[n]$ of the equaliser. In a fractionally spaced equaliser, the FF section is operated at a frequency higher than the symbol rate, which brings a number of advantages such as higher resolution, potentially shorter equalisers if the sampled channel contains fractional delays [3], or the capability to equalise channels with spectral zeroes [4].

Reasons why the FF section of an FS-DFE exhibits slow convergence will be reviewed in Sec. 2 based on properties of the general channel model $c[n]$. To improve on the convergence, Sec. 3 introduces an oversampled subband adaptive filter approach for the FF section, which exploits the prewhitening effect of the filter banks on the input signal [5]. The convergence behaviour of this subband FS-DFE is evaluated in Sec. 4 by comparing it against a standard FS-DFE algorithm under a severely distorting channel including spectral zeros. Conclusions are drawn in Sec. 5 . 


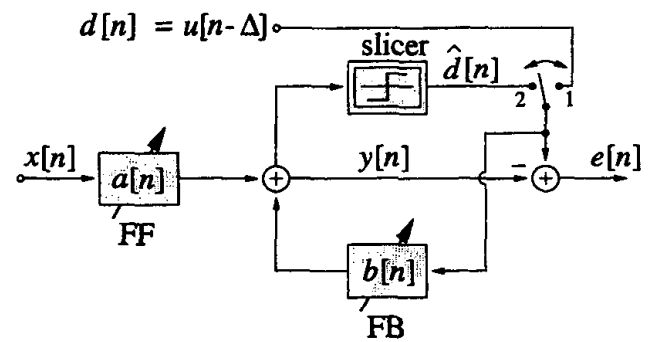

Fig. 2. Equaliser in (1) training and (2) decision feedback mode.

\section{Channel Model, Signal Properties, and LMS Convergence Issues}

This section focuses on signal properties, in particular spectral dynamics, which arise from characteristics of the transmission channel. It is assumed that the channel $c[n]$ is a baseband channel model, including filters for pulse shaping, modulation, the characteristics of the physical transmission medium, the demodulation, and the matched filter. The channel input $u[n]$ to the channel is here assumed to be nearly white, which can be achieved by appropriate scrambling of the symbols to be transmitted.

Pulse shaping is usually applied in order to bandlimit the transmitted signal, and a matched filter is placed on the receiving side. The system of concatenated transmit- and receive filters forms a Nyquist system popularly approximated by lowpass filters such as root-raised-cosine or Kingsbury filters [6]. If the channel output is sampled at a higher rate, i.e. twice the symbol rate, and in the absence of any channel distortion, this lowpass filter characteristic reflects the power spectral density (PSD) of the input signal $x[n]$ to the equaliser.

The convergence speed of LMS-type adaptive algorithms is inversely proportional to the eigenvalue spread of the covariance matrix of $x[n]$, which can be approximated by the ratio between maximum and minimum magnitude value of the PSD of the input signal $x[n]$ [7]. Obviously, the spectral dynamics imposed by the pulse shaping and matched filters on a $T / 2$-spaced input signal $x[n]$ are considerable, and the modes of convergence in the upper half of the spectrum are considerably slowed down.

Additional dynamics in the spectrum are introduced by the physical transmission channel. These dynamics become particularly sever when the channel exhibits spectral zeros resulting in deep notches in the PSD of $x[n]$. In this case, a direct $T$-spaced equalisation would most likely only boost the noise in the frequency

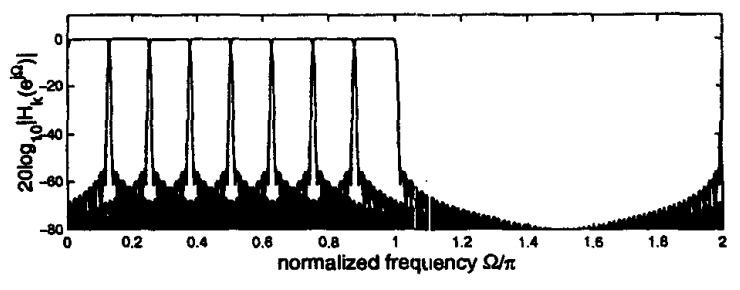

Fig. 3. Magnitude response of filter bank, only showing half the bands covering the spectrum $[0 ; \pi]$.

band of the spectral zero. Using a fractionally spaced equaliser, aliasing created in the decimation stage at the output of the FF section $a[n]$ can be exploited to fill the frequency gap created by a spectral zero [4].

\section{Subband Decorrelation for FS-DFE}

The general DFE structure in Fig. 2 contains two FIR filter sections. The input to the FF filter part is the channel output, which potentially exhibits strong spectral dynamics as introduced in the previous Sec. 2. Therefore, methods to decorrelate the input to the FF filter appear beneficial to increase the convergence speed of the overall DFE. For the FB section the input can be an explicit training signal $d[n]$, which is a delayed version of the transmitted signal $u[n]$. Alternatively, a desired signal $\hat{d}[n]$ can be estimated by thresholding the equaliser output $y[n]$ by the non-linear slicer of Fig. 2. Since the sequence of symbols is generally scrambled in order to achieve whiteness, decorrelation methods for the feedback section are not required [1].

The decorrelation method investigated here is based on a subbanding scheme prior to adaptive equalisation. Dividing the spectrum into a number of decimated frequency bands, the spectral dynamics within each so called subband are reduced compared to the fullband signal, yielding a reduced eigenvalue spread which in LMS-type adaptive filtering applications can be exploited for faster convergence. An example for a filter bank characteristic dividing the spectrum into $K=16$ subbands is shown in Fig. 3 . The filters shown have been modulated by a generalized discrete Fourier transform (GDFT, [8]) from a prototype lowpass filter. Besides the whitening effect, a second advantage lies in the generally reduced computational complexity due to the lower sampling rate of the decimated subband signals.

The proposed subband architecture for a fractionally $(T / 2)$ spaced equaliser is given in Fig. 4. Only the FF part is operated in the subband domain, whereby the oversampled input signal $x[n]$ is decomposed by an 


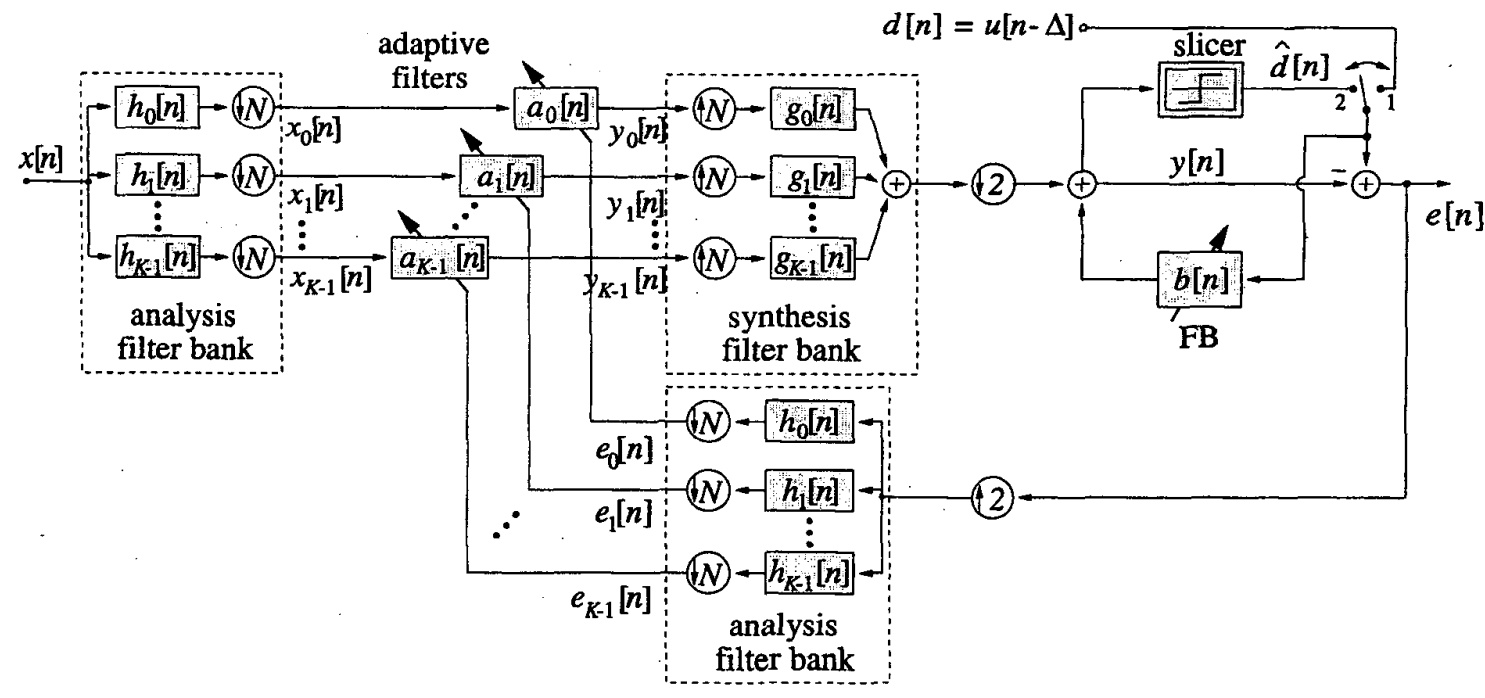

Fig. 4. Subband structure for FF part of $T / 2$-spaced Equaliser.

analysis filter bank. An independent subband adaptive filter is calculated in each subband, with the outputs being reconstructed to a fullband signal by a synthesis filter bank. To perform the error calculation at symbol rate, the synthesis filter output has to be decimated by a factor of two, which can be judiciously incorporated into the synthesis filter bank operation. The error calculation either for an equation error IIR or DFE algorithm - depending on the switch positions in Fig. 4 - has to take place at the symbol rate. This error is translated back into the subband domain by another analysis filter bank. The subbands are non-critically decimated by a factor $N<K$, whereby the oversampling ratio (OSR) $K / N$ is kept close to unity. The OSR is selected such that low alias levels arise in the subbands, since this limits the minimum mean square error (MMSE) performance of the adaptive filters [9], while still being small enough to ensure high computational efficiency [10].

Since for the DFE employs a non-linear decision device at the equaliser output, this system block cannot be translated into the subband domain or exchanged with linear system blocks such as the analysis or synthesis filter banks. Hence the subband architecture of Fig. 4 with an error calculation in the fullband has to be selected. Note however, that for an equation error IIR structure only, the desired signal could be decomposed by an analysis bank. Each subband would contain a two-channel adaptive filter updated by the according subband error signal. For compatibility with the DFE, we have restricted ourselves to the fullband error structure of Fig. 4. Due to the delay in the er- ror path between the adaptive filter outputs $y_{k}[n]$ and the subband errors $e_{k}[n]$, the adaptive filters have to be updated by a delay-LMS or delay-NLMS algorithm with somewhat inferior properties over standard LMS and NLMS adaptive filters [11].

The subband adaptive FF filter $a_{k}[n]$ in Fig. 4 are operated at an $N$ times slower rate compared to a fullband FS-DFE. For this reason, filters $a_{k}[n]$ can be selected approximately $N$ times shorter than the fullband FF filter to achieve similar modelling capabilities. Due to the reduced length and the lower update rate, the computational complexity of the subband device is generally decreased with respect to a fullband implementation. A judicious filter bank implementation is important and generally based on modulated filter banks [12]. Here, additional savings arise if the decimator $\downarrow 2$ in Fig. 4 is incorporated into the synthesis filter bank, and the upsampler $\uparrow 2$ into the analysis filter bank decomposing the error signal $e[n]$.

\section{Simulations and Results}

To demonstrate some properties of the presented subband FS-DFE, a dispersive channel with a delay spread of approximately 100 symbol periods and spectral zeroes has been selected. The channel $c[n]$ from Fig. 1 also includes Kingsbury filters extending over 5 symbol periods [6]. The overall magnitude response of the complex valued baseband channel model $c[n]$ of Fig. 1 is shown in Fig. 5. The output of this system is sampled at twice the symbol rate and fed into the FS-DFE 


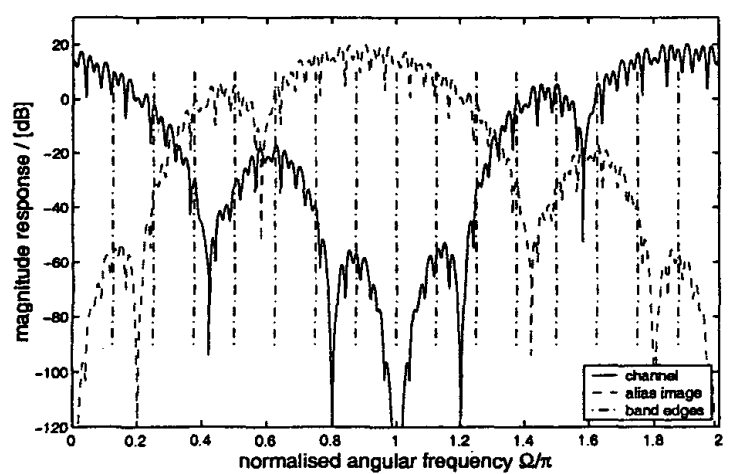

Fig. 5. Channel characteristics with receivetransmit filter and two additional 2 spectral zeroes.

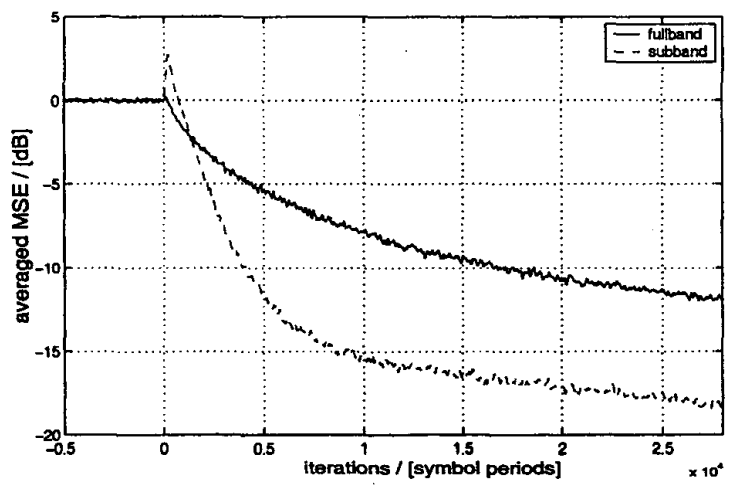

Fig. 6. Learning curves for fullband and subband $\mathrm{T} / 2$-spaced equalisers.

The subband FS-DFE uses $K=16$ channels (with band edges as indicated in Fig. 5) decimated by $N=$ 14 , and employs a length of the FF filter of $L_{\mathrm{FF}, \mathrm{s}}=40$. The fullband $T / 2$ spaced equaliser has a FF filter of length $L_{F F, f}=500$, while the feedback $(F B)$ filter is of identical dimension for both systems with $L_{\mathrm{FB}}=60$. In either case the delay of the equaliser is set such that the FF part almost only equalises the pre-cursor, and the feedback system the post-cursor.

\subsection{Convergence}

A first simulation was performed in the absence of channel noise with Gray coded 64-QAM as modulation mode. The updating algorithm for both fullband and subband FS equalisers used an NLMS algorithms (a delay-NLMS in the ilatter case), with a normalised

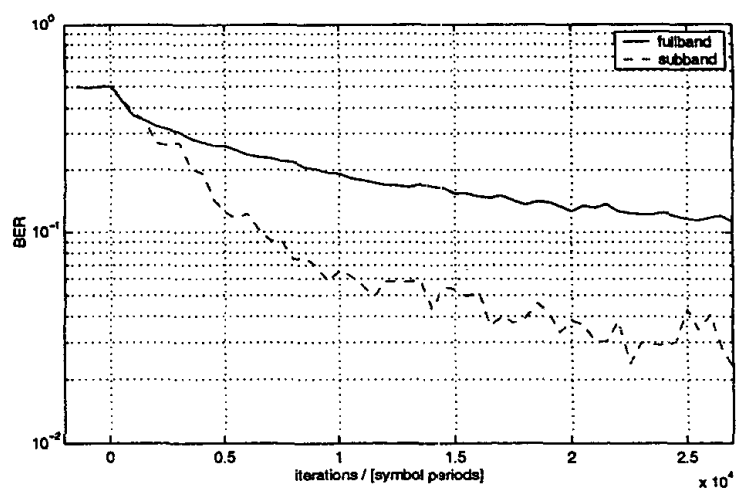

Fig. 7. Learning curves for fullband and subband $\mathrm{T} / 2$-spaced equalisers.

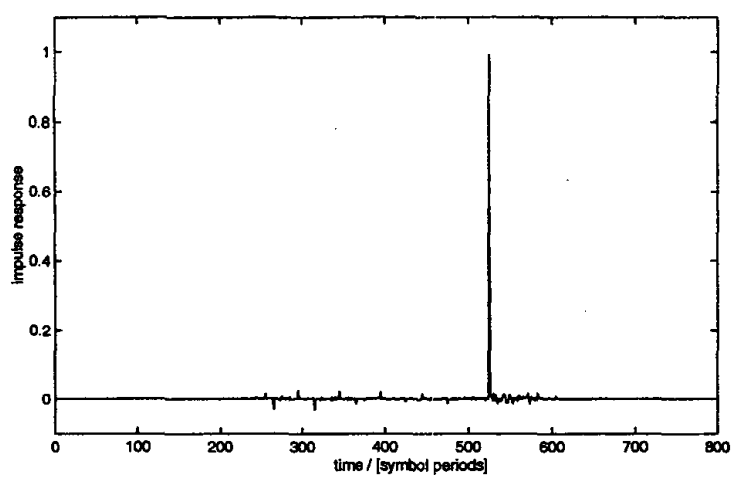

Fig. 8. Real part of the overall impulse response of channel and subband FS-DFE equaliser sampled at symbol rate.

step size of $\tilde{\mu}=0.4[7]$. The convergence speed of both equalisers is compared in Figs. 6 and 7 . The MSE curves in Fig. 6 are averaged over 20 trials and temporally smoothed. For both fullband and subband systems, adaptation is started at time $k=0$, and simulations run for $3 \cdot 10^{4}$ symbol periods. The subband FS equaliser adapts considerably faster than its fullband counterpart.

As a second performance inclicator, the overall transfer path of channel and equaliser is measured every 500 iteration during the simulation. For a bit stream input and the Gray coded 64-QAM, the resulting bit error rates (BER) over simulation time are presented in Fig. 7, again highlighting a superior convergence for the subband system. An example for the impulse response (sampled at symbol rate) for the overall system of channel and subband FS-DFE after $3 \cdot 10^{4}$ iterations 


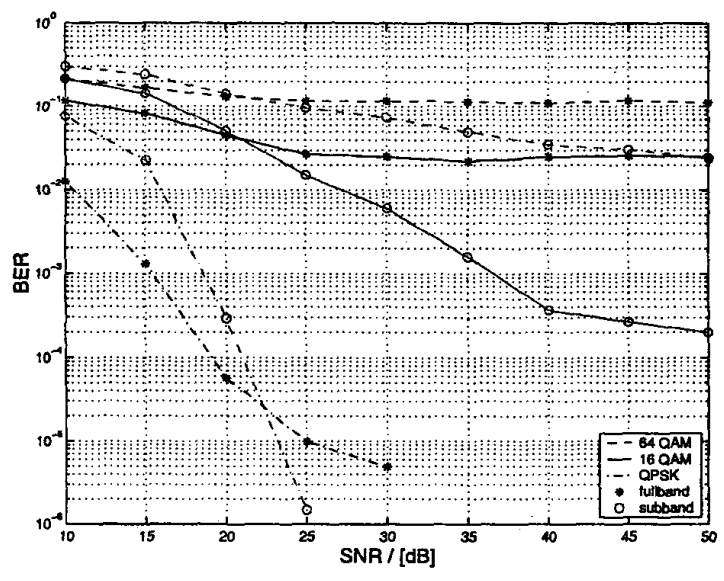

Fig. 9. Bit error rates after $3 \cdot 10^{4}$ iterations for both fullband and subband FS-DFE using different QAM constellations.

is shown in Fig. 8, where the pre-cursor and post-cursor are reasonably well equalised.

\subsection{Bit Error Rates}

A comparison between the fullband and subband FS-DFE is drawn for a noisy channel with various signal-to-noise ratios (SNR) at the equaliser input. The noise is normally distributed, and shaped by the matched filter in the receiver, i.e. exhibits a lowpass characteristic. Different Gray coded QAM schemes (QPSK, 16-QAM, 64-QAM) are employed to determine the achievable BER. The BER is based on the impulse response of the overall channel / equaliser system sampled at symbol rate after $3 \cdot 10^{4}$ iterations of adaptation.

The resulting BER for different QAM modulations and SNR are outlined in Fig. 9. For low SNR, the fullband FS equaliser attains consistently better BER performance for all modulation modes than the proposed subband architecture. For less noisy channels with an SNR above $20 \mathrm{~dB}$ however, the subband system achieves considerably better BER, which is mainly due to the fact that the proposed schemes is adapting faster within the allowed time interval of $3 \cdot 10^{4}$ iterations. This is clear from Fig. 7, to which the BER values for 64-QAM in Fig. 9 will tend for infinite SNR.

\section{Conclusions}

We have investigated an FS-DFE structure based on a subband decomposition of the feedforward part. This is achieved using oversampled filter banks, which results in a whitening or decorrelation of the input signal and hence offers faster convergence for LMS-type algorithms. Concerning the suitability of the proposed method, our initial results indicate that the subband FS-DFE can improve the convergence speed of the equaliser, particularly at high SNR where fullband FSDFEs have been found to converge slowly [1].

\section{References}

[1] M. Rupp, "On the Learning Behaviour of Decision Feedback Equalizers," in Asilomar Conference on Signals, Systems, and Computers, Monterey, CA, October 1999.

[2] John J. Shynk, "Adaptive IIR Filtering," IEEE Acoustics, Speech, and Signal Processing Magazine, vol. 6, no. 2, pp. 4-21, April 1989.

[3] K. D. Kammeyer, Nachrichtenübertragung, B.G.Teubner, Stuttgart, Germany, 1992.

[4] C. B. Papadias and D. T. M. Slock, "Fractionally Spaced Equalization of Linear Polyphase Channels and Related Blind Techniques Based on Multichannel Linear Prediction," IEEE Transactions on Signal Processing, vol. 47, no. 3, pp. 641-654, March 1999.

[5] W. Kellermann, "Analysis and Design of Multirate Systems for Cancellation of Acoustical Echoes," in Proc. IEEE International Conference on Acoustics, Speech, and Signal Processing, New York, 1988, vol. 5, pp. 2570-2573.

[6] Nick G. Kingsbury, "Transmit and Receive Filter for QPSK Signals to Optimise the Performance on Linear and Hard-Limited Channels," IEE Proceedings $F$, vol. 133, no. 4, pp. 345-355, July 1986.

[7] S. Haykin, Adaptive Filter Theory, Prentice Hall, Englewood Cliffs, 2nd edition, 1991.

[8] R. E. Crochiere and L. R. Rabiner, Multirate Digital Signal Processing, Prentice Hall, Englewood Cliffs, NJ, 1983.

[9] S. Weiss, R. W. Stewart, A. Stenger, and R. Rabenstein, "Performance Limitations of Subband Adaptive Filters," in European Signal Processing Conference, Rodos, Greece, September 1998, vol. III, pp. 12451248.

[10] S. Weiss and R. W. Stewart, On Adaptive Filtering in Oversampled Subbands, Shaker Verlag, Aachen, Germany, 1998.

[11] M. Rupp and R. Frenzel, "Analysis of LMS and NLMS Algorithms with Delayed Coefficient Update under the Presence of Spherically Invariant Processes," IEEE Transactions on Signal Processing, vol. SP-42, no. 3, pp. 668-672, March 1994.

[12] S. Weiss and R. W. Stewart, "Fast Implementation of Oversampled Modulated Filter Banks," IEE Electronics Letters, vol. 36, no. 17, pp. 1502-1503, August 2000 . 